\title{
Gyrokinetic investigation of the ASDEX Upgrade I-mode pedestal
}

\author{
K. Stimmel, ${ }^{1, a)}$ A. Bañón Navarro, ${ }^{1}$ T. Happel, ${ }^{1}$ D. Told, ${ }^{1}$ T. Görler, ${ }^{1}$ E. Wolfrum, ${ }^{1}$ \\ J.P. Martin Collar, ${ }^{1}$ R. Fischer, ${ }^{1}$ P.A. Schneider, ${ }^{1}$ F. Jenko, ${ }^{1}$ and The ASDEX Upgrade Team ${ }^{1}$ \\ 1) Max Planck Institute for Plasma Physics, Boltzmannstr. 2, 85748 Garching, \\ Germany
}

(Dated: January 29, 2020)

\begin{abstract}
Characterizing pedestal turbulence in the tokamak I-mode is a crucial step in understanding how particle and heat transport decouple during I-mode operation. This work models an ASDEX Upgrade I-mode discharge for the first time via linear and nonlinear gyrokinetic simulations with the GENE code. L-mode and I-mode regimes at two different pedestal locations are investigated. A microtearing mode which is not apparent in initial value linear L-mode simulations is found to dominate in I-mode simulations at both radial positions, and ion-scale instabilities are characterized for all four scenarios linearly. Computed nonlinear heat flux values approach experimental measurements with nominal input parameters in three of the four cases, and heat transport is found to be dominated by ion-scale electrostatic turbulence. Electrostatic potential oscillation frequencies, as well as potential-temperature and potential-density crossphases are compared linearly and nonlinearly, and agreement is found at wavenumber ranges corresponding with peaks in the simulated heat flux spectra at one radial position for L-mode and I-mode.
\end{abstract}

\section{INTRODUCTION}

The improved energy confinement regime (Imode) offers a route forward for future fusion reactors without the risk of edge localized modes (ELMs) which can deposit unacceptable heat loads to the divertor region. A promising mode of operation, the I-mode offers improved energy confinement while maintaining low confinement mode (L-mode)-like density profiles. ${ }^{1}$ The I-mode has been observed in Alcator C-mod, ${ }^{2-4}$ ASDEX Upgrade, ${ }^{1,5,6}$ and DIII-D, ${ }^{7,8}$ is the chosen regime for the future experimental reactor $\mathrm{ARC}^{9}$, and is an area of active research. ${ }^{1,2,5,6,9 ?-12}$ Stationary operation on different machines makes the I-mode an appealing regime for tokamaks. ${ }^{13,14}$

The mechanisms which allow for the decoupling of density and temperature profiles in the I-mode have not yet been fully understood, although common characteristics in

\footnotetext{
a)Electronic mail: karl.stimmel@ipp.mpg.de
}

both C-Mod and ASDEX-Upgrade I-mode discharges include heightened temperature profiles simultaneous with low density profiles, and the weakly coherent mode (WCM), a pedestal mode that is observed to be electrostatic in ASDEXUpgrade I-mode plasmas. ${ }^{10}$

While experimental diagnostics have given much insight into the nature of the I-mode, and other gyrokinetic simulations have expanded theoretical understanding of turbulent transport in I-mode scenarios, a clear understanding of turbulent transport in the tokamak pedestal has not yet been developed for the I-mode. White et al. showed that the I-mode is closer than the L-mode to the respective critical ion temperature gradient responsible for experimentally relevant heat fluxes in core simulations of C-Mod using GYRO. ${ }^{15} \mathrm{X}$. Liu et al. performed global nonlinear GENE simulations for C-Mod I-mode scenarios and related the WCM to an ion temperature gradient (ITG) mode, but simulations were limited in wavenumber resolution in the binormal direction due to computational demand. ${ }^{16}$ Creely et al. performed GYRO simulations for C-Mod 
and found that multiscale effects may be less important for I-mode than L-mode, ${ }^{17}$ Z.X. Liu showed that the WCM is linearly unstable to drift Alfven wave instabilities and the resistive ballooning mode with BOUT ++ simulations, ${ }^{4}$ and Mikkelsen et al. demonstrated electrostatic verification of GENE and GYRO simulations in the I-mode core for C-mod parameters, although heat fluxes disagreed with experimental measurements. ${ }^{18}$ This work shows gyrokinetic simulation results from the GENE $\operatorname{code}^{19,20}$ for ASDEX-Upgrade I-mode pedestal scenarios for the first time, using available experimental measurements as direct simulation inputs. Linear instabilities are characterized, nonlinear heat transport is compared with experimental values, and linear-nonlinear comparisons are drawn in an attempt to elucidate the decoupling between temperature and density profiles in the ASDEX Upgrade I-mode as well as to explore potential for future reduced gyrokinetic models. This paper is structured in the following way: in Sec. II, an overview of the AUG discharge \#30865 is given. In Sec. III the GENE code is briefly outlined in the context of this work, and the corresponding assumptions for numerical simulation are qualified. In Sec. IV the linear results from the GENE simulations are presented, and in Sec. V, nonlinear GENE results are presented and comparisons are drawn with the experiment. Linear-nonlinear comparisons are given in Sec. VI, and discussion of future possibilities for gyrokinetic I-mode work is presented in Sec. VII along with a summary and discussion of results.

\section{CHARACTERIZATION OF THE I-MODE DISCHARGE}

ASDEX Upgrade discharge \#30865 features both L-mode and I-mode phases throughout the discharge and has previously been investigated in detail in Ref. 12. The discharge utilized upper single null magnetic geometry to achieve an 'unfavorable' magnetic configuration, where the ion $\nabla B \times B$ drift points away from the active $\mathrm{X}$ point, thus allowing a higher power threshold to transit into H-mode. ${ }^{3}$ For ASDEX Upgade shot \#30865, the toroidal magnetic field was $-2.48 \mathrm{~T}$ on axis with a plasma current of 1 MA.

Fig. 1 illustrates the time-resolved experimental measurements for AUG shot \#30865. Electron cyclotron resonance heating (ECRH), neutral beam injection (NBI), and heating and radiative power losses at the separatrix excluding the divertor region are shown in Fig. 1(a). NBI blips every $0.5 \mathrm{~s}$ allow for ion temperature measurements via charge exchange recombination spectroscopy (CXRS) ${ }^{21}$ without impacting the plasma proportion. The plasma is dominantly heated by ECRH which is increased at $3 \mathrm{~s}$ to $1.7 \mathrm{MW}$ and at $3.5 \mathrm{~s}$ to $2.2 \mathrm{MW}$, allowing the plasma to enter a weak I-mode at $3.18 \mathrm{~s}$ and fully enter I-mode after $3.5 \mathrm{~s}$. The experimental total heat flux, calculated by subtracting the power radiated inside the separatrix excluding the divertor region from the total input heating power $\left(P_{\text {Tot }}-P_{\text {rad,core }}\right)$ is $1.763 \mathrm{MW}$ for L-mode and 2.026 MW for I-mode.

Fig. 1(b) illustrates that NBI blips fail to excite an improved confinement state at $2 \mathrm{~s}$ and $2.5 \mathrm{~s}$, but shortly after the NBI blip and increased ECRH heating at $3 \mathrm{~s}$, the plasma begins to enter a weak I-mode. Note that from $3 \mathrm{~s}$ to $3.18 \mathrm{~s}$, the plasma is still in L-mode as illustrated by the semi-stationary H98 $(y, 2)$ factor $^{22}$ in Fig. 2 and by Fig. 1(d) from previous investigation in Ref. 12. The evolution from L-mode to I-mode is shown in Fig. 1(c), and Fig. 1(d) illustrates the electron temperature calculated using integrated data analysis (IDA) with bayesian statistics $^{23}$ at the two radial positions investigated. In both L-mode and I-mode, the electron plasma beta, $\beta_{e}$, is generally low but increases from L-mode to I-mode for both sampled radial positions. L-mode temperature and density profiles are sampled at $3.11 \mathrm{~s}$, consistent with previous investigations, ${ }^{12}$ and an I-mode phase with temperature $(T)$ and density $(n)$ profiles is sampled at $3.80 \mathrm{~s}$. Electron density and temperature profile data are available for both 3.11 $\mathrm{s}$ and $3.80 \mathrm{~s}$ seconds, while ion temperature profiles, dependent on CXRS measurements, are instead sampled at $3.0 \mathrm{~s}$ and $4.0 \mathrm{~s}$, which are the times corresponding to the NBI blips closest to 


\begin{tabular}{l|llllllllll}
\hline \hline Phys. Input & $\rho_{\text {tor }}$ & $T_{e}$ & $n_{e}$ & $q_{0}$ & $\hat{s}$ & $\beta_{e} \times 10^{-3}$ & $Z_{\text {eff }}$ & $\omega_{T e}$ & $\omega_{T i}$ & $\omega_{n}$ \\
\hline L-mode & \multirow{2}{*}{0.95} & 212 & 1.51 & 4.16 & 4.10 & 0.209 & 1.306 & 12.27 & 7.42 & 8.40 \\
I-mode & & 343 & 1.76 & 4.22 & 4.09 & 0.402 & 1.407 & 16.70 & 12.40 & 10.86 \\
\hline L-mode & \multirow{2}{*}{0.98} & 133 & 1.16 & 4.89 & 7.34 & 0.101 & 1.306 & 13.72 & 12.78 & 7.80 \\
I-mode & & 166 & 1.24 & 4.94 & 7.18 & 0.137 & 1.407 & 25.69 & 18.72 & 10.95 \\
\hline \hline
\end{tabular}

Table I. Summary of relevant GENE physics input parameters. Here $\rho_{\text {tor }}$ is the radial position, $T_{e}$ is the electron temperature at a given position in $\mathrm{eV}, n_{e}$ is the electron density in $10^{19} \mathrm{~m}^{-3}, q_{0}$ is the safety factor, $\hat{s}=\left(\rho_{\text {tor }} / q\right)\left(d q / d \rho_{\text {tor }}\right)$ is the magnetic shear, $\beta_{e}$ is the ratio of thermal electron pressure to magnetic pressure, $Z_{\text {eff }}$ is the effective plasma charge estimate, and $\omega_{T e, T i, n}$ are the normalized density gradients for $T_{e}, T_{i}$ and $n$, respectively.

\subsection{1 and 3.8 seconds, respectively.}

To characterize the I-mode pedestal, two radial locations are investigated at $\rho_{\text {tor }}=0.95$ and $\rho_{\text {tor }}=0.98$ to allow for differences in the quantities in Tab. I to be considered. The following simulations utilize temperature profiles taken from the experiment for both ions and electrons, as well as a radially constant estimate of the effective charge, $Z_{\text {eff }}$, taken from CXRS and Bremmstrahlung measurements on ASDEX Upgrade. This estimate is found to vary widely from a minimum of 1.2 with Bremmstrahlung measurements giving $Z_{\text {eff }}$ of about 1.6 for most of the discharge. In this work, $Z_{\text {eff }}$ is modelled to be slightly higher in I-mode to be consistent with trends observed in CXRS and Bremmstrahlung measurements; the values used are presented in Table I. Electron density and temperature profiles are generated using IDA and are shown in Fig. 3(a) and (b), respectively. Despite using IDA profiles, a large degree of experimental uncertainty exists for the edge profiles. Ion density profiles are calculated with $Z_{\text {eff }}$ by enforcing quasi-neutrality and assuming a globally charge-neutral plasma. Ion temperature profiles are displayed in Fig. 3 (c). In Fig. 3 (d),(e), and (f), the normalized gradients used for density, electron temperature, and ion temperature are shown for L-mode and Imode, respectively. Here the normalized gradients are defined as $\omega_{k}=-(a / k)\left(d k / d \rho_{\text {tor }}\right)$ where $k$ is density or temperature. The radial coordinate, $\rho_{\text {tor }}$, is defined as $\rho_{\text {tor }}=$ $\sqrt{\Phi_{\text {tor }} / \Phi_{\text {tor,sep }}}$ with toroidal flux label $\Phi$, and $a=\sqrt{\left(\Phi_{\text {tor }, \text { sep }} /\left(\pi B_{0}\right)\right)}$ is the minor radius. The separatrix is located at $\rho_{\text {tor }}=1$ by this definition.

Three-species linear simulations utilize Boron impurity, background ion, and electron density profiles, while two-sepcies nonlinear simulations were computationally limited to background ion density profiles that are equivalent to the electron density profiles. Density profiles remain similar between L-mode and I-mode, while temperature profiles for both electrons and ions increase in the pedestal region during I-mode. Although density profile gradients were self-consistently evaluated for threespecies linear simulations and two-species nonlinear simulations, differences in gradient values are less than $0.03 \%$. Ion temperature profiles were consistently manually fitted with the available CXRS data. Fig. 3(d-f) show the normalized density and temperature scale lengths, respectively. At both radial positions, the temperature gradient increase is greater than the density gradient increase from L-mode to Imode. At $\rho_{\text {tor }}=0.95(0.98)$, the density gradient increases from L-mode and I-mode by $0.29 \%(0.40 \%)$, while the electron temperature gradient increases by $36 \%(87 \%)$ and the ion temperature gradient increases by $67 \%(46 \%)$.

\section{NUMERICAL MODELLING}

In the following, turbulent transport is modelled with the GENE code. GENE utilizes a five-dimensional phase-space grid consisting of three spatial and two velocity- 

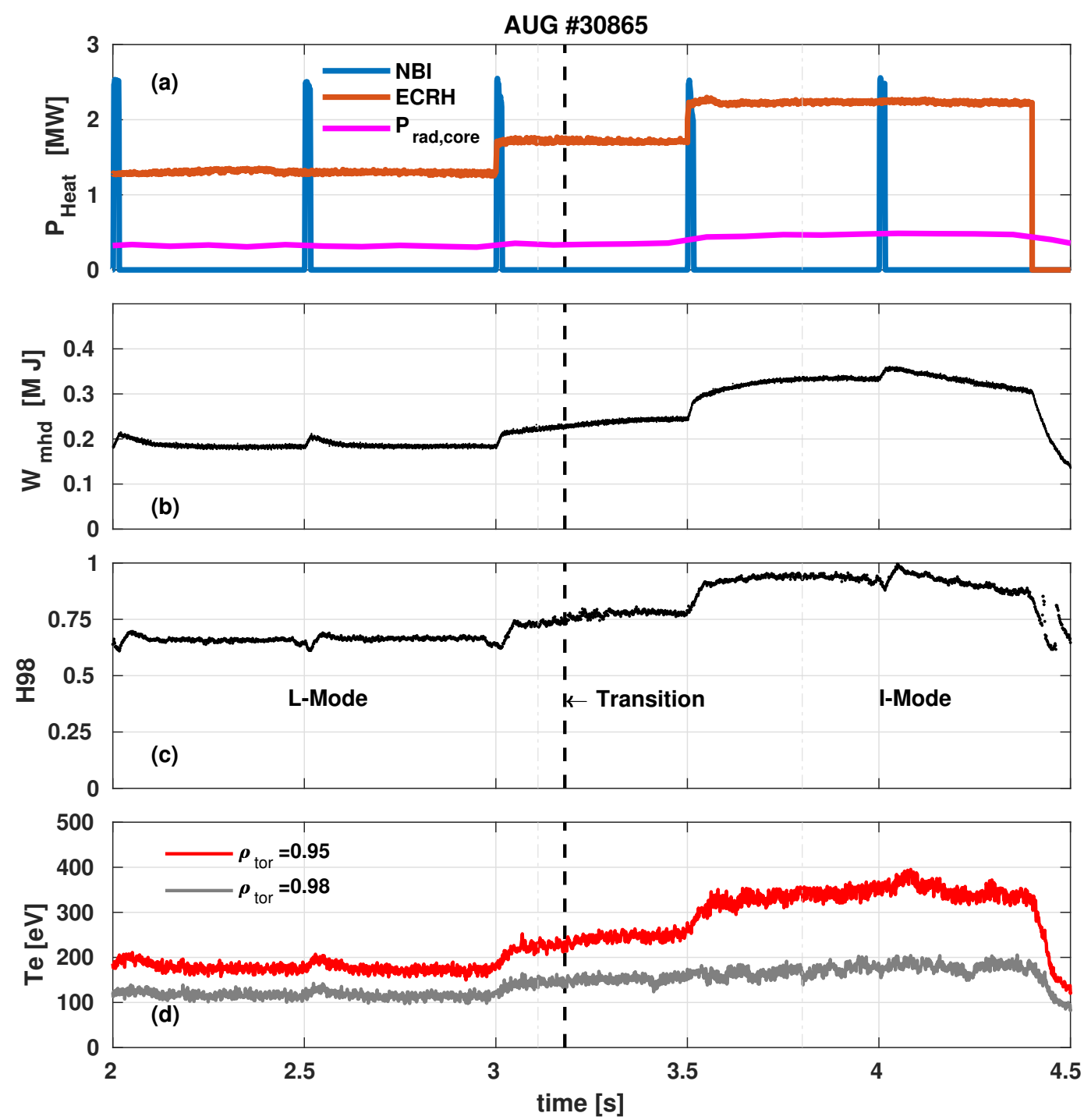

Figure 1. Experimental timetrace for AUG \#30865 from 2 s to 4.5 s. a)Heating power, dominated by electron cyclotron resonance heating $(\mathrm{ECRH}, \mathrm{red})$, power radiated from the core, $\left(\mathrm{P}_{\text {rad,core }}\right.$, magenta), and beam blips from neutral beams (NBI, blue) for diagnostic purposes. b) Total stored energy. c) Confinement enhancement H98. d) Integrated data analysis (IDA) electron temperature time traces at $\rho_{\text {tor }}=0.95$ and $\rho_{\text {tor }}=0.98$.

space dimensions. ${ }^{20}$ The radial, bi-normal, field aligned, parallel velocity, and magnetic moment coordinates are labelled as $x, y, z, v_{\|}$, and $\mu$, respectively. In this work, $\rho_{\mathrm{s}}$ is the reference ion or electron gyroradius for ion and electron-scale simulations, respectively, and the gyroradius to 


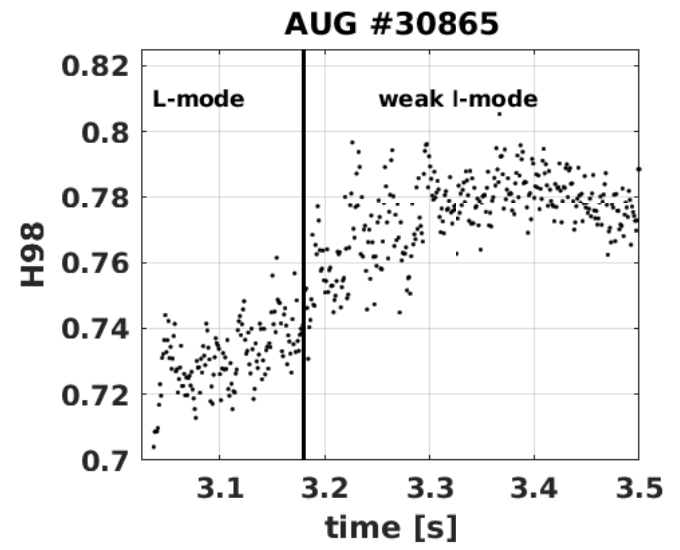

Figure 2. Experimental confinement enhancement H98 $(y, 2)$ immediately following an NBI blip and ECRH increase at $3 \mathrm{~s}$.

tokamak minor radius ratio is $\rho^{*}=\rho_{\mathrm{s}} / a$. Linear growth rates are normalized by $c_{s} / a$, where $c_{s}=\sqrt{T_{e} / m_{\mathrm{i}}}, T_{e}$ is the electron temperature, and $m_{i}$ is the ion mass. The binormal wavenumber is defined in GENE as $k_{y}$ and is normalized by $\rho_{s}$. All linear and nonlinear simulations presented are in local flux tube geometry, and include electromagnetic effects. The equilibria inputs for GENE are calculated using the CLISTE $\operatorname{code}^{24,25}$ for both time points under investigation.

Two time points are sampled corresponding to L-mode and I-mode, and each time point is further sampled at radial positions of $\rho_{\text {tor }}$ $=0.95$ and 0.98 for a total of four scenarios. For each scenario, linear local, and nonlinear local simulations are carried out. Linear simulations with periodic boundary conditions are performed over a range of toroidal mode numbers as a precursor to nonlinear investigation by identifying important unstable modes. Nonlinearly, ion- and electron-scale (adiabatic ions) simulations are carried out. Multi-scale interactions cannot be disregarded a priori, but are left to future work due to vast computational requirements and due to findings outlined in Sec. V that suggest most effects can be captured with ion-scale simulations. Flux tube simulations at these radial positions with peri-
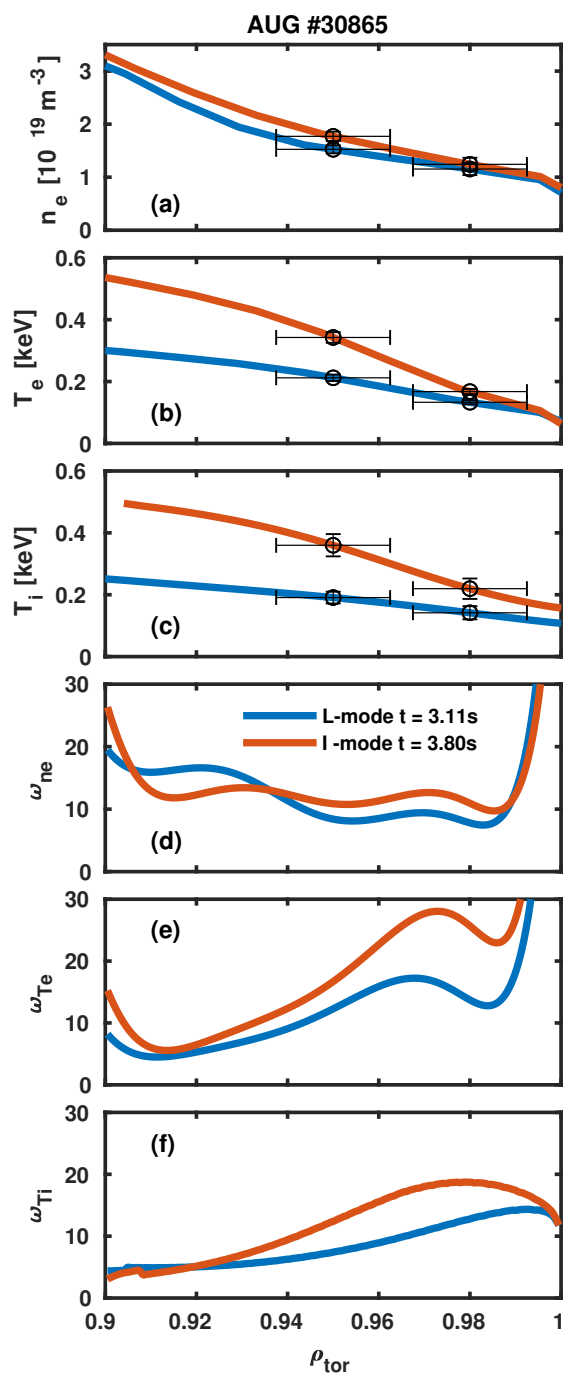

Figure 3. Experimental profiles for AUG shot \#30865 used as computational input. L-mode data is sampled at $3.11 \mathrm{~s}$, and I-mode data is sampled at $3.80 \mathrm{~s}$.

odic boundary conditions proved unfeasible due to the immense resolution and unrealistic box sizes needed to allow for numerical convergence in the parallel component of the magnetic vector potential, $A_{\|}$. For this reason, the nonlinear simulations are performed with Dirichlet boundary conditions and a Krook-type heating 
operator described in Ref. 20 to capture larger structures while allowing for computational feasibility. Nonlinear simulations use buffer zones that utilize the outer $10 \%$ of the simulation domain are implemented to damp unphysical temperature and density profile variations close to the edge of the simulation domain. Ultimately these findings demonstrate the potential of global simulations to more accurately model the pedestal region in steep gradient scenarios. Periodic boundary conditions are adequate for nonlinear electron scale flux tube simulations due to the smaller spatial scales. Radial electric field shearing is included for ion-scale nonlinear simulations by shifting the radial Fourier mode grid in time as outlined in Ref. 26.

\section{LINEAR INSTABILITY ANALYSIS}

Linear, local, initial value, flux tube simulations are performed for both the L-mode and Imode scenarios at two radial positions to determine the fastest growing instability for a range of toroidal wavenumbers. The growth rates are checked for convergence by increasing the numerical resolution of the simulations until a difference less than $5 \%$ is observed in the growth rates. The linear simulations have a resolution of $N_{x} \times N_{v_{\|}} \times N_{\mu}=17 \times 64 \times 16$ and $60<N_{z}<200$. Here, $N_{x}, N_{z}, N_{v_{\|}}$, and $N_{\mu}$ are the number of grid points in the radial coordinate, parallel coordinate, parallel velocity, and magnetic moment grids, respectively. The high $\mathrm{z}$ resolution is necessary to resolve low growth rates and ballooning structures in both scenarios due to strongly shaped flux surfaces at $k_{y} \rho_{s}<0.1$ and in some select cases for $k_{y} \rho_{s}>1$.

Linear growth rates and frequencies are investigated across a wide $k_{y} \rho_{s}$ range. Linear growth rates for L-mode and I-mode at the two radial positions are plotted in Fig. 4. An ion-scale peak exists for all four scenarios at $k_{y} \rho_{s}<1$ and an electron scale peak appears at $k_{y} \rho_{s}>100$. While it is necessary to perform nonlinear multiscale simulations to fully outline multiscale effects, the difference in magnitude of the linear ion-scale and electron-scale linear growth rate peaks suggest that ion and electron scale simulations can be decoupled based on previous work. ${ }^{27-29}$ Namely, if $\gamma_{e} / \gamma_{i} \gg \sqrt{\left(m_{i} / m_{e}\right)}$ where $\sqrt{\left(m_{i} / m_{e}\right)} \sim 60.6$, then multiscale effects are likely to be important. For the cases presented here, all $\gamma_{e} / \gamma_{i}$ do not exceed the square root of the mass ratio.

At $\rho_{\text {tor }}=0.95$ an additional intermediate ETG instability range exists around $k_{y} \rho_{s}=5$ to 10. An ion-scale instability analysis is shown in Fig. 5 as ion-scale heat transport is shown to dominate in Sec. V.

\section{A. Linear ion-scale analysis}

Figure $5(\mathrm{a}, \mathrm{b})$, linear growth rates for L-mode and I-mode are shown for $\rho_{\text {tor }}=0.95$ and 0.98 , respectively, with corresponding microtearing mode (MTM), trapped electron mode (TEM), ion temperature gradient (ITG), and electron temperature gradient (ETG) instabilities identified with corresponding markers. In Fig. 5 $(\mathrm{c}, \mathrm{d})$, the linear frequencies corresponding with the dominant linear instabilities are plotted for L-mode and I-mode for each radial position under investigation.

The MTM is identified by its parallel mode structure and electromagnetic dominated heat flux. At low $k_{y} \rho_{s}$ in Fig. 5 (a-d), higher growth rates were produced with the I-mode parameters, and a distinct tearing-parity electromagnetic instability is found indicated by star symbols, while the L-mode parameters produced low or zero growth rate. The real, imaginary, and absolute components of the tearing parity structure at low I-mode $k_{y} \rho_{s}$, shown in Fig. 6, illustrate an asymmetric potential mode structure and a symmetric magnetic vector potential mode structures consistent with an MTM. ${ }^{30}$ The difference in amplitude for each peak in Fig. 6 stems from the up-down asymmetric equilibrium. This shape is largely a result of the upper-single-null configuration used for this Imode discharge. ${ }^{12}$

Comparing the ratio of electromagnetic and electrostatic heat flux for a given linear simulation can give an indication of which type of 
instability and which species is predominantly responsible for electrostatic or electromagnetic heat flux. The MTM is further characterized by the ratio of linear electromagnetic to electrostatic heat flux. At a radial position of $\rho_{\text {tor }}=0.98$, the heat flux ratio is dominated by electron electromagnetic heat flux from $k_{y} \rho_{s}$ $=0.05$ to 0.06 and further inside the pedestal at $\rho_{\text {tor }}=0.95$ this range broadens from $k_{y} \rho_{s}$ $=0.0524$ to 0.0824 , consistent with the MTM ranges in Fig. 5 (c,d). Electron electromagnetic heat flux dominates at the aforementioned wavenumber ranges in I-mode scenarios for linear Dirichlet boundary condition calculations as well. Although the MTM occurs only in I-mode scenarios, it is likely unrelated to the WCM since the WCM has not been observed to have a measurable electromagnetic component experimentally on ASDEX Upgrade.

The dominant instability at the ion-scale peak around $k_{y} \rho_{s}=0.3$ is found to be TEM for all cases as shown in Fig. 5 (a-d). Increasing the density gradient is found to have a stabilizing effect, further suggesting TEM from $k_{y} \rho_{s}$ $=0.1$ to 1 . Heat flux in this regime is dominated by electron electrostatic heat flux with a negative frequency corresponding to an electron dominated instability. At high $k_{y} \rho_{s}$, an ETG mode dominates as evidenced by negative frequencies and electron electrostatic dominated heat transport. An intermediate ITG regime is found at $\rho_{\text {tor }}=0.95$ as shown in Fig. 5 (c), however the growth rate corresponding to the positive ITG frequencies is relatively low compared to the other growth rates. Some positive frequencies are also found at $\rho_{\text {tor }}=0.98$ shown in Fig. 5 (d), corresponding with ITG, but this small intermediate range is limited.

The linear instability analysis outlines the importance of ion scale simulations, suggests that electromagnetic effects are relevant, and distinguishes between the two radial positions investigated with a linear ITG range which only occurs further inside the pedestal. The following section corroborates these linear findings nonlinearly.

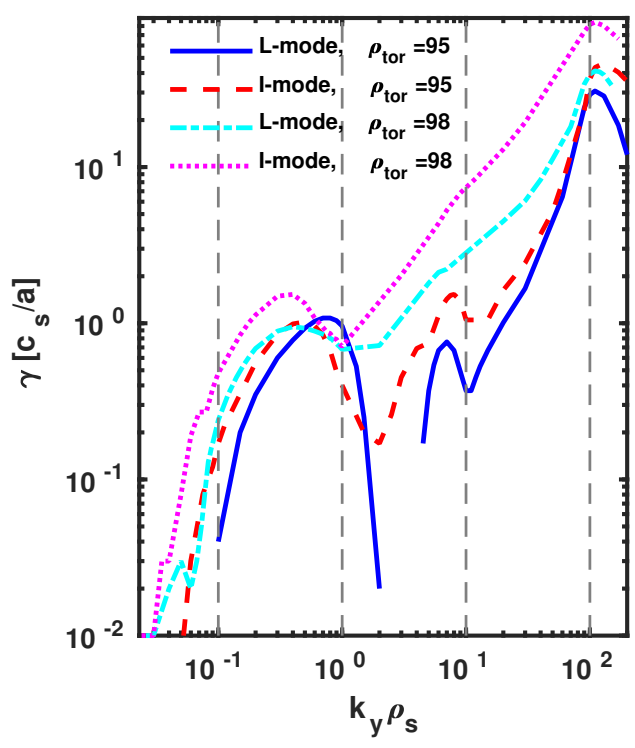

Figure 4. Linear, local, $k_{y} \rho_{s}$ scan for ASDEX Upgrade shot \#30865 at 3.11 and $3.80 \mathrm{~s}$. Converged growth rates, $\gamma$, are plotted against $k_{y} \rho_{s}$ for L-mode and I-mode at radial positions $\rho_{\text {tor }}=0.95$ and 0.98 .

\section{B. Neoclassical calculations}

In addition to nonlinear ion- and electronscale simulations, neoclassical simulations are also performed. Neoclassical transport is contained as a subset of the gyrokinetic equations solved in GENE. ${ }^{31,32}$ This is achieved in GENE by reducing the gyrokinetic model to a zeroth-order equation fulfilled by a Maxwellian distribution function. Neoclassical simulations have been shown to reproduce electron energy flux and ion energy flux to within $\sim 15 \%$ and $\sim 30 \%$, respectively, when comparing to the full Fokker-Planck model. ${ }^{33}$ Neoclassical transport is greater in I-mode scenarios, but composed only a portion of the total heat flux, with the greatest fraction being $5.3 \%$ of the total heat flux in the I-mode case at $\rho_{\text {tor }}=0.98$ as shown in Fig. 7. 

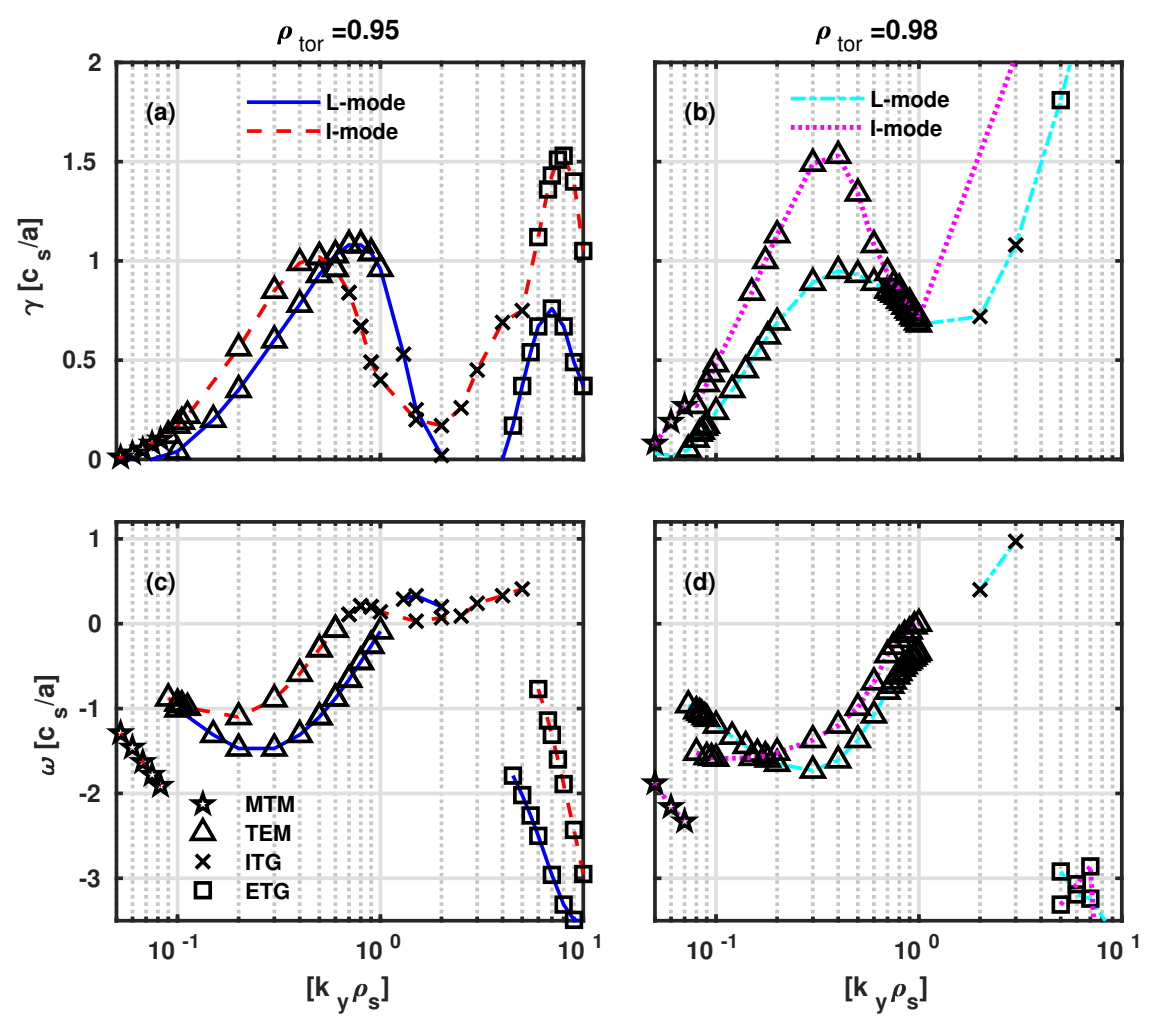

Figure 5. Linearly converged growth rates $(\gamma)$ and frequencies $(\omega)$ obtained from GENE for four scenarios for a wide range of $k_{y} \rho_{s}$. Growth rates for L- and I-mode, in a) blue and red at $\rho_{\text {tor }}=0.95$, and b) in teal and magenta at $\rho_{\text {tor }}=0.98$. Frequencies for L- and I-mode in c) blue and red at $\rho_{\text {tor }}=0.95$, and d) teal and magenta at $\rho_{\text {tor }}=0.98$. Microtearing modes $(\mathrm{MTM})$ are identified with stars, trapped electron modes (TEM) are identified with triangles, ion temperature gradient (ITG) modes are marked as crosses and electron temperature gradient (ETG) modes with square boxes.

\section{NONLINEAR GENE SIMULATIONS}

Having identified $k_{y} \rho_{s}$ ranges of interest through linear simulations, nonlinear simulations are performed separately at ion and electron scales. Heat flux, particle transport, and other quantities are determined by timeaveraging over the quasi-stationary state for each simulation unless otherwise stated. Potential oscillation frequencies are obtained by performing a Fourier transform in time over the quasi-stationary state. For each scenario, $\mathrm{E} \times \mathrm{B}$ shearing is included from available experimen- tal data. Results shown in figures are without shearing included unless stated otherwise.

\section{A. Ion-scale simulations}

Ion-scale nonlinear simulations in the pedestal regime are computationally demanding due to the high numerical resolution needed to resolve structures generated from steep gradients. The standard number of numerical resolution points, $N$, used for nonlinear ion-scale simulations is 


\begin{tabular}{|c|c|c|c|c|c|c|c|c|}
\hline \multirow[t]{2}{*}{ Heat Transport } & \multirow[t]{2}{*}{$\rho_{\text {tor }}$} & \multicolumn{3}{|c|}{$\rho_{i}$ scale } & \multirow{2}{*}{$\frac{\rho_{e} \text { scale }}{\left[\cdot 10^{-3}\right]}$} & \multirow[t]{2}{*}{ neocl. } & \multicolumn{2}{|c|}{ Heat Flux sum } \\
\hline & & ${ }_{\text {Ion }}^{\mathrm{E}}$ & $e^{-}$ & $\begin{array}{c}\text { EM } \\
\text { Ion } e^{-}\end{array}$ & & & sim. & exp. \\
\hline$\overline{\mathrm{L}-\text { mode }}$ & 0.95 & 0.50 & 1.41 & $\begin{array}{ll}0.00 & 0.01\end{array}$ & $\overline{1.1}$ & 0.04 & 1.96 & 1.77 \\
\hline $\mathrm{I}-$ mode & 0.95 & 11.67 & 16.52 & -0.151 .74 & 9.5 & 0.19 & 29.83 & 2.03 \\
\hline $\mathrm{L}-$ mode & 0.98 & 0.25 & 0.46 & $0.00 \quad 0.00$ & 0.90 & 0.03 & 0.74 & 1.77 \\
\hline $\mathrm{I}-$ mode & 0.98 & 0.57 & 1.37 & -0.010 .00 & 14.8 & 0.11 & 2.05 & 2.03 \\
\hline
\end{tabular}

Table II. Summary of simulated time-averaged nonlinear heat fluxes averaged over the flux tube area and experimental reference values in MW.
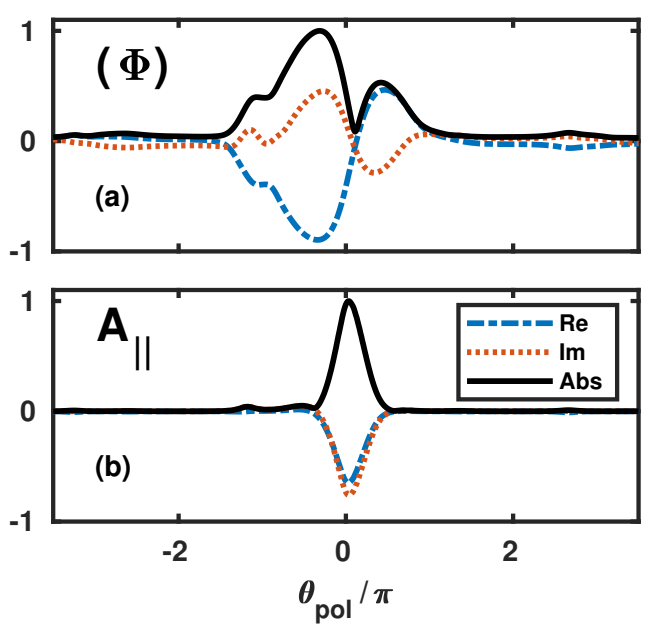

Figure 6. Parallel mode structure for a linear Imode MTM at $k_{y} \rho_{s}=0.06$ at $\rho_{\text {tor }}=0.95$. a) Normalized amplitude of electrostatic potential $\Phi$ and b) parallel magnetic vector potential $A_{\|}$.

$N_{x} \times N_{z} \times N_{v_{\|}} \times N_{\mu}=512 \times 60 \times 32 \times 16$ with varying $68<N_{k y}<108$ depending on the grid size needed to resolve the flux spectra. The minimum ion-scale wavenumber, $k_{y, \text { min,ion }}$ is set to 0.022 or less depending on the case to fully resolve the heat flux spectra. Nonlinear box size and radial resolution convergence tests were performed using different radial domain sizes to determine a box size of $\mathrm{Lx}=200 \rho_{\mathrm{s}}$ for nonlinear simulations. The box size used is ultimately a compromise to ensure convergence while allowing for computational feasibility and the preservation of the local limit. Shearing induced by the nominal values of available toroidal rotation data have been included to study the effects from $\mathrm{E} \times \mathrm{B}$ shearing. The ion-scale simulations are carried out with two species, again due to immense computational demand necessary for three-species simulations. Despite only using two species, charge effects are still included in GENE via the $Z_{\text {eff }}$ parameter which affects collisionality. Including active impurities in nonlinear simulations is left to future work.

Table II lists the computed time-averaged heat flux values for the ion-scale nonlinear, electron-scale nonlinear, and neoclassical heat fluxes. Ion-scale simulation results are divided into electrostatic (ES) and electromagnetic (EM) channels and further subdivided into ion and electron channels. Electron-scale and neoclassical heat flux sums contribute only a fraction of the total heat fluxes. A total sum of the simulated heat fluxes is presented next to the experimentally measured value in the final two columns.

In all scenarios, heat transport is dominantly electrostatic, with a greater contribution of the heat flux coming from the electron rather than the ion channel. The I-mode case at $\rho_{\text {tor }}=0.95$ significantly overpredicts the experimental heat flux value and yields positive frequency fluctuations rather than negative frequencies as predicted by linear simulations.

This discrepancy is explained by a nonlinear shift in the critical $\beta_{e}$ threshold necessary to enter the kinetic ballooning mode (KBM) limit consistent with the previous studies in Ref. 34. Linearly, at $k_{y} \rho_{s}=0.15$, a significant 
increase in growth rate at the peak is observed as $\beta_{e}$ is increased from the nominal value to $\beta_{e}=10^{-3}$. Nonlinearly, this increase in heat flux occurs slightly below the nominal $\beta_{e}$ value at $\sim \beta_{e}=3 \cdot 10^{-4}$, an order of magnitude lower than the linear predicted $\beta_{e}$ threshold. Lowering $\beta_{e}$ by $50 \%$ and reducing both temperature gradients by $30 \%$ reduces the heat flux by $83 \%$ relative to the nominal value for the I-mode case at $\rho_{\text {tor }}=0.95$ and allows for quasilinear potential frequency agreement to be recovered. The nominal I-mode parameters at $\rho_{\text {tor }}=0.98$ are farther removed from this $\beta_{e}$ threshold nonlinearly, thus precluding the possibility for a significant overprediction near nominal parameters. Furthermore the nonlinear shift observed here occurs at values of $\beta_{e}$ similar to previous investigations. ${ }^{34}$ Finally, including $\mathrm{E} \times \mathrm{B}$ shearing reduces heat flux further, however a full exploration of $\mathrm{E} \times \mathrm{B}$ shearing values within the error bars is left to future work.

Heat fluxes for the three cases which approach experimental heat flux values with nominal parameters are plotted in Fig. 7. Electron electrostatic heat transport, shown in light blue, composes the majority of the heat transport, followed by ion electrostatic and neoclassical transport in dark blue and black, respectively.

At $\rho_{\text {tor }}=0.95$, the particle to heat diffusivity ratio increases from L-mode to I-mode as expected from experimental intuition, however the simulated heat flux for the I-mode case at $\rho_{\text {tor }}=0.95$ does not agree with the experimental value. At $\rho_{\text {tor }}=0.98$, the particle to heat diffusivity ratio decreases from L-mode to I-mode, contrary to experimental intuition. This indicates that the I-mode particle transport is reduced relative to the total heat flux. One explanation is that the particle to heat diffusivity ratio merely follows the density to temperature gradient ratio at $\rho_{\text {tor }}=0.98$. Inclusion of $\mathrm{E} \times \mathrm{B}$ shearing from nominal parameters did not alter this relationship; stronger $\mathrm{E} \times \mathrm{B}$ shearing may be necessary to modify the particle to heat diffusivity ratio.

The ratio of electron to ion heat flux is driven by temperature gradients as evidenced in Tab. III. For a given radial position, as the elec-

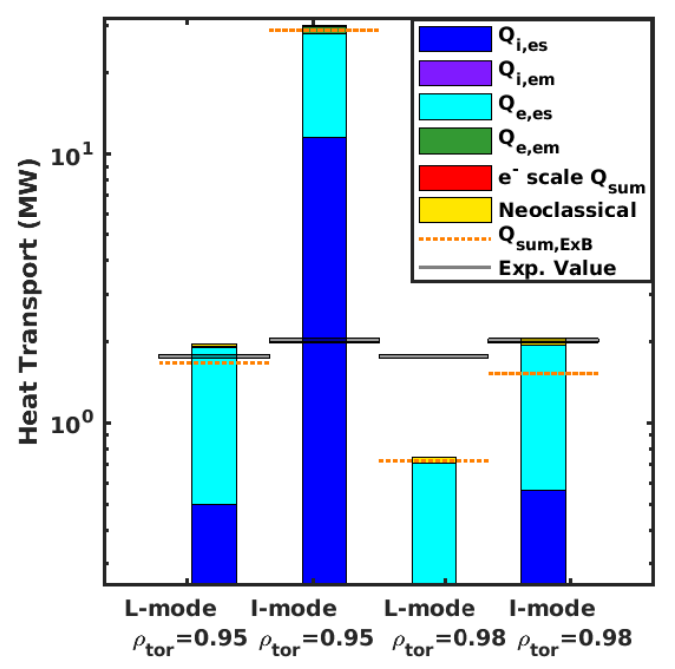

Figure 7. Nonlinear time-averaged heat flux values for ion/electron ion-scale electrostatic (dark blue/light blue), ion/electron ion-scale electromagnetic (purple/green), electron scale sum (red), and neoclassical (yellow) simulations for three scenarios in MW. The red dashed lines show the total heat flux if nominal $\mathrm{E} \times \mathrm{B}$ shearing is included for ionscale nonlinear simulations. Experimental heat flux values are overplotted in gray.

tron to ion temperature gradient decreases, the ratio of electron to ion heat flux subsequently decreases.

To further investigate the differences between the L-mode and I-mode cases, two additional 'hybrid' cases are carried out where L-mode profiles and equilibria are simulated with I-mode gradients. It is found that artificially imposing I-mode gradients only increases the heat flux to $8.7 \%$ and $60.6 \%$ of the original I-mode heat flux at $\rho_{\text {tor }}=0.95$ and 0.98 , respectively. Despite heat flux being dominantly electrostatic, electromagnetic considerations via the $\beta_{e}$ parameter and equilibria are crucial. 


\begin{tabular}{l|llll}
\hline \hline Ratio & $\rho_{\text {tor }}$ & $\omega_{T e} / \omega_{T i}$ & $Q_{e} / Q_{i}$ & $D / \chi$ \\
\hline L - mode & \multirow{2}{*}{0.95} & 1.65 & 2.84 & 0.28 \\
I - mode & & 1.35 & 1.59 & 0.33 \\
\hline L - mode & \multirow{2}{*}{0.98} & 1.07 & 1.84 & 0.27 \\
I - mode & & 1.37 & 2.45 & 0.23 \\
\hline
\end{tabular}

Table III. Relevant nonlinear ratios for the investigated cases. Radial position is given in $\rho_{\text {tor }}$, $\omega_{T e} / \omega_{T i}$ is the ratio of electron to ion temperature gradient, $Q_{e} / Q_{i}$ is the ratio of electron to ion heat flux, and $D / \chi$ is the ratio of particle diffusivity to heat diffusivity.

\section{B. Electron-scale simulations}

A set of simulations at high $k_{y} \rho_{e}$ are also performed to ascertain the contribution to the overall heat flux from electron-scale turbulence. The resolution used for nonlinear electron-scale simulations is $N_{x} \times N_{z} \times N_{v_{\|}} \times N_{\mu}=256 \times$ $80 \times 32 \times 16$ and $160<N_{k y}<168$. The lowest electron-scale wavenumber is set to $k_{y, \min , e}$ $=0.02$ for all nonlinear electron-scale simulations to fully resolve the heat flux spectra peak. Despite electron-scale simulations being well resolved in $k_{y} \rho_{s}$, the electron heat flux only contributes $5 \%$ of the total heat flux for the nominal I-mode case at $\rho_{\text {tor }}=0.95$ and less than $1 \%$ for all other cases.

Considering that other I-mode studies have shown that electron-scale transport can be significant, ${ }^{16}$ additional simulations with destabilized gradients are also investigated to assess the robustness of the low electron-scale heat transport. By decreasing the density gradient by $20 \%$ and increasing the electron temperature gradient by $20 \%$, the heat flux in all four scenarios increases, but only to $0.07 \mathrm{MW}$ at most in the I-mode cases.

\section{CROSSPHASE ANALYSIS}

Of particular interest in I-mode studies is how density profiles remain L-mode like while temperature profiles steepen. Determining whether the decoupling of particle and heat transport is related to the fluctuating quantities' relationship to each other is therefore a logical point of investigation. Crossphases provide valuable information about which quantities are responsible for transport and how various fluctuating quantities are related to one another, and can be compared linearly and nonlinearly. Fig. 8 shows the I-mode crossphases at $\rho_{\text {tor }}=0.98$, where the potential-perpendicular temperature fluctuation $\left(\Phi \times T_{\perp}\right)$ and potential-density fluctuation $(\Phi \times n)$ crossphases for ions and electrons are defined in the same way as in Ref. 35:

$$
\left\langle\nu\left[\tilde{\Phi}, \tilde{T}_{\perp, j}\right]\right\rangle_{k_{x}}\left(k_{y}\right)=\frac{\sum_{k_{x}} \arg \left[\tilde{\Phi}\left(k_{x}, k_{y}\right) / \tilde{T}_{\perp, j}\left(k_{x}, k_{y}\right)\right] W\left(k_{x}, k_{y}\right)}{\sum_{k_{x}} W\left(k_{x}, k_{y}\right)}
$$

where $W\left(k_{x}, k_{y}\right)=\left|\tilde{\phi}\left(k_{x}, k_{y}\right)\right|\left|\tilde{T}_{\perp, j}\left(k_{x}, k_{y}\right)\right|$ is an amplitude dependent weighting factor, $\langle. .\rangle_{k_{x}}$ indicates an average over all $k_{x}$ modes, and $j$ is the species index. The $\Phi \times n$ crossphase is nearly centered around the phase angle of zero, for both ions and electrons. Although the $\Phi \times T_{\perp, i}$ crossphase is centered around zero, the $\Phi \times T_{\perp, e}$ crossphase is shifted slightly out of phase. The crossphases for the L-mode case at $\rho_{\text {tor }}=0.98$, omitted for brevity, mimic the Imode crossphases, with the exception that the $\Phi \times T_{\perp, e}$ crossphase is shifted slightly away from zero phase angle and toward higher $k_{y} \rho_{s}$. Interestingly, despite differences in the input profiles and resulting heat fluxes, the crossphases for Lmode and I-mode are similar, illustrating that the separation of density and temperature profiles cannot be explained by out-of-phase fluctuations alone. The inclusion of $\mathrm{E} \times \mathrm{B}$ shearing has a marginal effect on results shown in Fig. 8.

Linear and nonlinear simulation results are compared to ascertain the potential for gyrokinetic quasi-linear modelling in the far edge. The simulations at $\rho_{\text {tor }}=0.98$ are analyzed here, due to the fact that they most accurately reproduce heat flux as an L-mode/I-mode pair.

To assess the viability of linear predictions for nonlinear transport, a crossphase analysis comparing linear and nonlinear simulations is performed. I-mode results are shown here for conciseness, but L-mode results have similar char- 


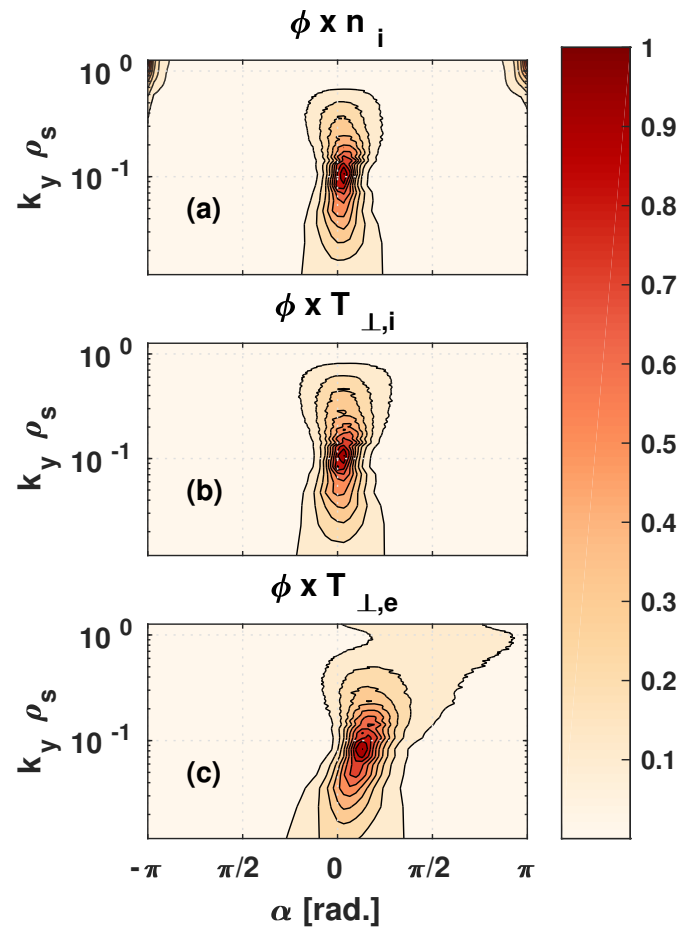

Figure 8. Normalized crossphase for the nonlinear simulation at I-mode, $\rho_{\text {tor }}=0.98$. Amplitudes are weighted for each $k_{y} \rho_{s}$ mode. Crossphase angle $\alpha$ is given on the x-axis. a) $\Phi \times n_{i}$, b) $\Phi \times T_{i, \perp}$, c) $\Phi \times T_{e, \perp}$.

acteristics at $\rho_{\text {tor }}=0.98$. Figure 9 illustrates the relationship between the linear and nonlinear I-mode crossphases observed for $\Phi \times n$ and $\Phi \times T_{\perp, i, e}$ for both electrons and ions. The corresponding ion and electron heat flux spectra $Q_{i, e}$ in GENE normalized units of $\left[c_{s} n_{e} T_{e}\left(\rho^{*}\right)^{2}\right]$ are overplotted in Fig. 9(a) and (b). It can be seen that I-mode ion and electron $\Phi \times n$ crossphases are in reasonable agreement linearly and nonlinearly. At low and high $k_{y} \rho_{i}$, linear and nonlinear crossphases for $\Phi \times T_{\perp, i, e}$ do not agree as closely, however at regions of high nonlinear transport, agreement is notably better. This suggests that at $k_{y} \rho_{s}$ where nonlinear transport is high, linear crossphase predictions are more likely to be accurate for both ions and electrons.
The inclusion of nominal $\mathrm{E} \times \mathrm{B}$ values has little effect on the linear-nonlinear crossphase relationship shown in Fig. 9. The region of peaking heat flux and better quasi-linear agreement corresponds with the TEM range found linearly in Fig. 5. Electron electromagnetic heat flux peaks at a $k_{y} \rho_{s}=0.03$ to 0.05 , which is slightly lower than the linear MTM range of $k_{y} \rho_{s}=0.05$ to 0.07 , suggesting that the MTM is washed out by stronger instabilities or is entirely absent nonlinearly.

Figure 10 shows the frequencies for the Imode scenario at $\rho_{\text {tor }}=0.98$ together with the experimentally expected WCM range from Ref. 12. The color scale here indicates the normalized nonlinear amplitude for each $k_{y} \rho_{s}$. The WCM for this discharge is expected to occur at $k_{y} \rho_{s}=0.2$, which is slightly higher than the electrostatic heat flux peak. Here no frequency amplitude maxima enter the WCM range. Global simulations or the inclusion of impurities may be necessary for higher frequencies to emerge based on the findings of previous work which had success in some cases matching the WCM. ${ }^{16}$ As with the cross-phases agreement, the linear-nonlinear frequency maxima agreement improves at $k_{y} \rho_{s}$ where the heat flux spectra peaks nonlinearly.

\section{SUMMARY AND DISCUSSION}

The results shown in this work show clear differences in L-mode and I-mode scenarios for ASDEX Upgrade shot \#30865 and illustrate the various relationships between these scenarios at different radial positions. Heat transport is dominantly electrostatic, however electromagnetic effects via the $\beta_{e}$ parameter are important, demonstrating that differences in the plasma pressure profile and equilibrium are key I-mode characteristics.

Linear simulations identify instabilities which are predominantly electrostatic in nature and together with nonlinear results show that ionscale turbulence is of importance in such pedestal scenarios. Previous work which utilized experimental inputs from Alcator C-Mod 

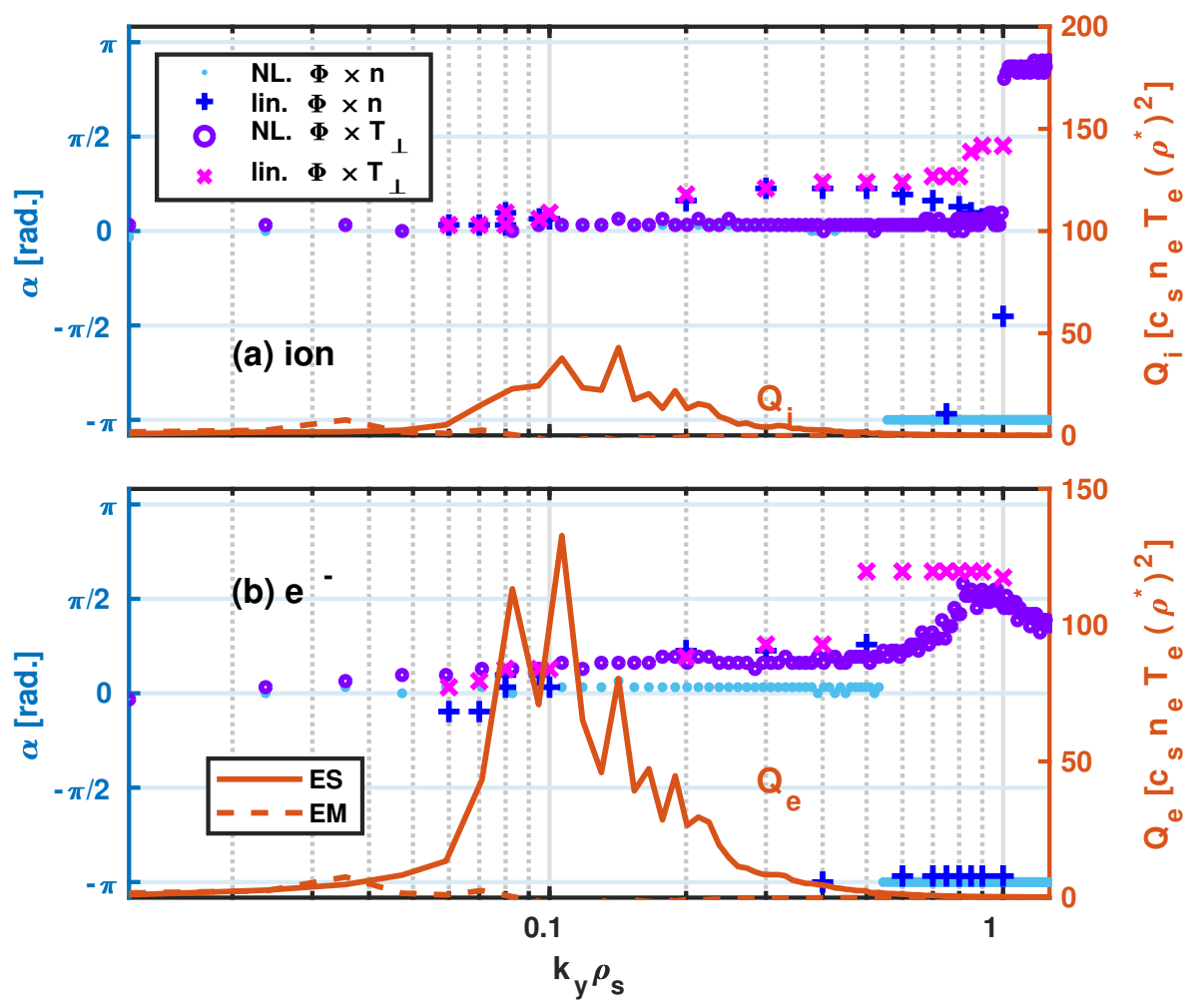

Figure 9. Nonlinear (NL.) and linear (lin.) simulation maximum crossphase ion and electron amplitude for $\Phi \times n$ and $\Phi \times T_{\perp}$ for AUG shot \#30865 at 3.80 seconds (I-mode). Nonlinear time-averaged electrostatic (ES) and electromagnetic (EM) heat flux spectra are overlaid for reference.

found that local electron-scale simulations contributed a significant portion of the total nonlinear heat flux ${ }^{16}$ opposed to findings presented here, suggesting that the differences in electronscale heat fluxes arise from differences in experimental input. Electromagnetic effects must be carefully considered as evidenced by shortcomings in matching I-mode heat fluxes with hybrid scenarios and the nonlinear $\beta_{e}$ threshold shift in the I-mode case at $\rho_{\text {tor }}=0.95$.

Nonlinear heat fluxes approach the experimental values in three of the four scenarios modelled. Results from this work suggest that heat flux from electron scales is secondary to transport generated at ion scales. Electrostatic ionscale heat transport is found to dominate, and
$\mathrm{E} \times \mathrm{B}$ shearing reduces the heat flux in all cases. For some cases, sensitivity is observed upon changing the simulation box size, demonstrating that global simulations may be necessary to simultaneously damp pedestal structures and allow for the full interaction between core and edge. The local nonlinear simulations presented here are limited by computational feasibility and are therefore a first step towards understanding the ASDEX Upgrade I-mode pedestal. Evidence of a WCM range was not found despite electrostatic heat flux spectra peaking at a similar wavenumber range as the WCM. Resolving the WCM may require additional degrees of realism such as inclusion of impurities, global simulations, ${ }^{16}$ or a full scan of $\mathrm{E} \times \mathrm{B}$ shearing 


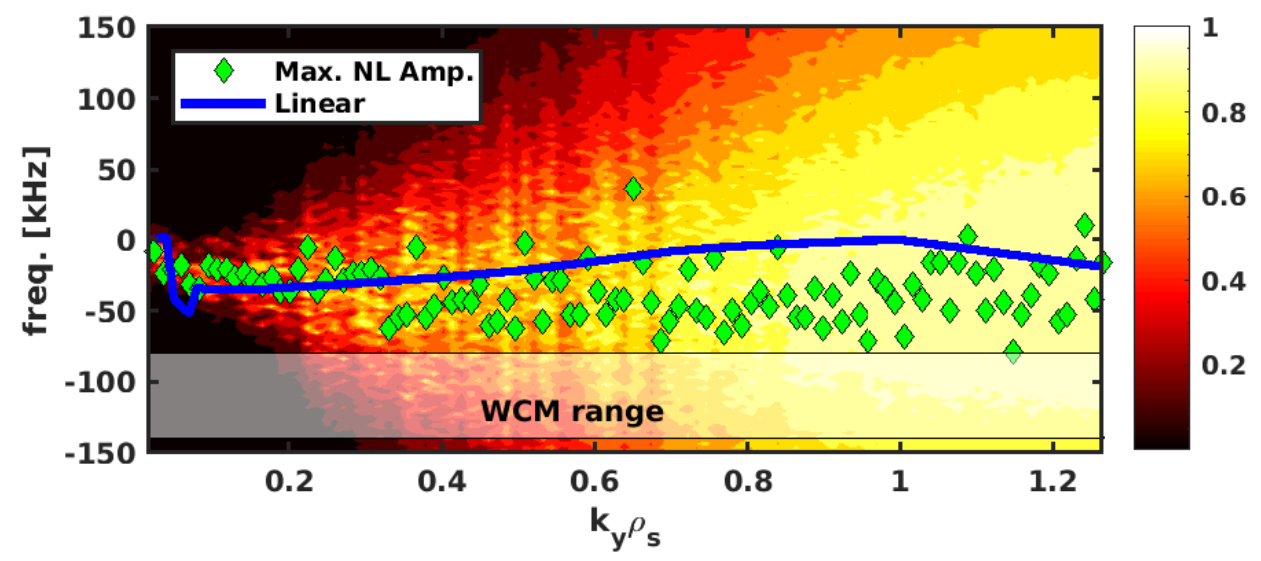

Figure 10. Nonlinear frequency amplitude spectra for the I-mode scenario at $\rho_{\text {tor }}=0.98$. Frequency maxima for each $k_{y} \rho_{s}$ are plotted via color gradient, with maximum amplitude points marked with diamonds. Linear maximum frequency amplitude is overplotted in blue.

values. The computational expense of pedestal simulations stems from the high numerical resolution needed to resolve steep-gradient scenarios; this limits current investigations and motivates the study of reduced models in the pedestal.

A crossphase analysis indicates that similar crossphases are found in both L-mode and Imode. Linear and nonlinear frequencies and crossphases show better agreement at wavenumbers that correspond to nonlinear heat flux spectra peaks for cases where the nonlinear heat flux approaches the experimental value. This provides potential motivation for the verification and development of future quasilinear models. Further exploration of I-mode cases with additional degrees of realism in a global context are a topic of further study.

\section{ACKNOWLEDGEMENTS}

The author would like to thank P. Crandall, N. Bonanomi, D. Hatch, P. Manz, K. Höfler, M. Dunne, and the GENE team at IPP Garching for insightful discussions regarding experimental and GENE-related analyses. A large fraction of the simulations carried out in this work have been performed on supercomputers at the Max Planck Computing and Data Facility (MPCDF, formerly known as RZG). This research also used resources of the National Energy Research Scientific Computing Center (NERSC), a U.S. Department of Energy Office of Science User Facility operated under Contract No. DE-AC02-05CH11231. This work has been carried out within the framework of the EUROfusion Consortium and has received funding from the Euratom research and training program 2014-2018 and 2019-2020 under grant agreement No 633053. The views and options expressed herein do not necessarily reflect those of the European Commision.

\section{REFERENCES}

${ }^{1}$ T. Happel, P. Manz, F. Ryter, P. Hennequin, A. Hetzenecker, G. D. Conway, L. Guimarais, C. Honoré, U. Stroth, and E. Viezzer, Nuclear Fusion 56 (2016), 10.1088/0029-5515/56/6/064004.

${ }^{2}$ A. E. Hubbard, D. G. Whyte, R. M. Churchill, I. Cziegler, A. Dominguez, T. Golfinopoulos, J. W. Hughes, J. E. Rice, I. Bespamyatnov, M. J. Greenwald, N. Howard, B. Lipschultz, E. S. Marmar, M. L. Reinke, W. L. Rowan, and J. L. Terry, Physics of Plasmas 18, 56115 (2011). 
${ }^{3}$ A. Hubbard, D. Whyte, R. Churchill, A. Dominguez, J. Hughes, Y. ma, E. Marmar, Y. Lin, M. Reinke, and A. White, Nuclear Fusion 52, 114009 (2012).

${ }^{4}$ Z. X. Liu, X. Q. Xu, X. Gao, A. E. Hubbard, J. W. Hughes, J. R. Walk, C. Theiler, T. Y. Xia, S. G. Baek, T. Golfinopoulos, D. Whyte, T. Zhang, and J. G. Li, Physics of Plasmas 23, 120703 (2016), https://doi.org/10.1063/1.4972088.

${ }^{5}$ T. Happel, P. Manz, F. Ryter, M. Bernert, M. Dunne, P. Hennequin, A. Hetzenecker, U. Stroth, G. D. Conway, L. Guimarais, C. Honoré, and E. Viezzer, Plasma Physics and Controlled Fusion 59 (2017), 10.1088/0741-3335/59/1/014004.

${ }^{6}$ F. Ryter, R. Fischer, J. Fuchs, T. Happel, R. McDermott, E. Viezzer, E. Wolfrum, L. Barrera Orte, M. Bernert, A. Burckhart, S. da Graça, B. Kurzan, P. McCarthy, T. Pütterich, W. Suttrop, M. Willensdorfer, and t. ASDEX Upgrade Team, Nuclear Fusion 57 (2017), 10.1088/0029-5515/57/1/016004.

${ }^{7}$ A. Hubbard, T. Osborne, F. Ryter, M. Austin, L. B. Orte, R. Churchill, I. Cziegler, M. Fenstermacher, R. Fischer, S. Gerhardt, R. Groebner, P. Gohil, T. Happel, J. Hughes, A. Loarte, R. Maingi, P. Manz, A. Marinoni, E. Marmar, R. McDermott, G. McKee, T. Rhodes, J. Rice, L. Schmitz, C. Theiler, E. Viezzer, J. Walk, A. White, D. Whyte, S. Wolfe, E. Wolfrum, and Z. Yan, 56, 086003 (2016).

${ }^{8}$ A. Marinoni, J. Rost, M. Porkolab, A. Hubbard, T. Osborne, A. White, D. Whyte, T. Rhodes, E. Davis, D. Ernst, and K. Burrell, Nuclear Fusion 55, 093019 (2015).

${ }^{9}$ B. N. Sorbom, J. Ball, T. R. Palmer, F. J. Mangiarotti, J. M. Sierchio, P. Bonoli, C. Kasten, D. A. Sutherland, H. S. Barnard, C. B. Haakonsen, J. Goh, C. Sung, and D. G. Whyte, Fusion Engineering and Design 100, 378 (2015), arXiv:1409.3540.

${ }^{10}$ D. Whyte, A. Hubbard, J. Hughes, B. Lipschultz, J. Rice, E. Marmar, M. Greenwald, I. Cziegler, A. Dominguez, T. Golfinopoulos, N. Howard, L. Lin, R. McDermott, M. Porkolab, M. Reinke, J. Terry, N. Tsujii, S. Wolfe, S. Wukitch, and Y. Lin, Nuclear Fusion 50, 105005 (2010).

${ }^{11}$ I. Cziegler, P. H. Diamond, N. Fedorczak, P. Manz, G. R. Tynan, M. Xu, R. M. Churchill, A. E. Hubbard, B. Lipschultz, J. M. Sierchio, J. L. Terry, and C. Theiler, Physics of Plasmas 20, 055904 (2013), https://doi.org/10.1063/1.4803914.

${ }^{12}$ P. Manz, P. Lauber, V. Nikolaeva, T. Happel, F. Ryter, G. Birkenmeier, A. Bogomolov, G. Conway, M. Manso, M. Maraschek, D. Prisiazhniuk, and E. Viezzer, Nuclear Fusion 55, 083004 (2015).

${ }^{13}$ A. E. Hubbard, S. G. Baek, D. Brunner, A. J. Creely, I. Cziegler, E. Edlund, J. W. Hughes, B. Labombard, Y. Lin, Z. Liu, E. S. Marmar, M. L. Reinke, J. E. Rice, B. Sorbom, C. Sung, J. Terry, C. Theiler, E. A. Tolman, J. R. Walk, A. E. White, D. Whyte, S. M. Wolfe, S. Wukitch, and X. Q. Xu, Nuclear Fusion 57 (2017), 10.1088/1741-4326/aa8570.
${ }^{14}$ T. Happel, M. Griener, D. Silvagni, S. Freethy, P. Hennequin, F. Janky, P. Manz, D. Prisiazhniuk, F. Ryter, M. Bernert, D. Brida, T. Eich, M. Faitsch, L. Gil, L. Guimarais, A. Merle, D. Nille, J. Pinzón, B. Sieglin, and E. Viezzer, Nuclear Materials and Energy 18, 159 (2019).

${ }^{15}$ A. E. White, N. T. Howard, A. J. Creely, M. A. Chilenski, M. Greenwald, A. E. Hubbard, J. W. Hughes, E. Marmar, J. E. Rice, J. M. Sierchio, C. Sung, J. R. Walk, D. G. Whyte, D. R. Mikkelsen, E. M. Edlund, C. Kung, C. Holland, J. Candy, C. C. Petty, M. L. Reinke, and C. Theiler, Physics of Plasmas 22 (2015), 10.1063/1.4921150.

${ }^{16} \mathrm{X}$. Liu, PhD Thesis, University of Texas at Austin (2018).

${ }^{17}$ A. J. Creely, N. T. Howard, P. Rodriguez-Fernandez, N. Cao, A. E. Hubbard, J. W. Hughes, J. E. Rice, A. E. White, J. Candy, G. M. Staebler, G. D. Conway, S. J. Freethy, and C. Sung, Physics of Plasmas 24 (2017), 10.1063/1.4977466.

${ }^{18}$ D. R. Mikkelsen, N. T. Howard, A. E. White, and A. J. Creely, Physics of Plasmas 25 (2018), $10.1063 / 1.5018741$

${ }^{19}$ F. Jenko, W. Dorland, M. Kotschenreuther, and B. N. Rogers, Physics of Plasmas 7, 1904 (2000), https://doi.org/10.1063/1.874014.

${ }^{20}$ T. Görler, X. Lapillonne, S. Brunner, T. Dannert, F. Jenko, F. Merz, and D. Told, Journal of Computational Physics 230, 7053 (2011).

${ }^{21}$ E. Viezzer, T. Pütterich, R. Dux, and R. M. McDermott, Review of Scientific Instruments 83, 103501 (2012), https://doi.org/10.1063/1.4755810.

${ }^{22}$ ITER Physics Basis Editors and ITER Physics Expert Group Chairs and Co-Chairs and ITER Joint Central Team and Physics Unit, Nuclear Fusion 39, 2137 (1999).

${ }^{23}$ R. Fischer, C. J. Fuchs, B. Kurzan, W. Suttrop, and E. Wolfrum, Fusion Science and Technology 58, 675 (2010).

${ }^{24}$ P. J. McCarthy, Physics of Plasmas 6, 3554 (1999), https://doi.org/10.1063/1.873630.

${ }^{25}$ P. J. McCarthy, Plasma Physics and Controlled Fusion 54, 015010 (2011).

${ }^{26}$ G. Hammett, W. Dorland, N. Loureiro, and T. Tatsuno, (2006), 48th APS DPP Conference.

${ }^{27}$ T. Görler and F. Jenko, Phys. Rev. Lett. 100, 185002 (2008).

${ }^{28}$ T. Görler and F. Jenko, Physics of Plasmas 15, 102508 (2008), https://doi.org/10.1063/1.3006086.

${ }^{29}$ F. Jenko, Journal of Plasma and Fusion Research SERIES 6, 11 (2004).

${ }^{30}$ D. Hatch, D. Told, F. Jenko, H. Doerk, M. Dunne, E. Wolfrum, E. Viezzer, and M. Pueschel, Nuclear Fusion 55, 063028 (2015).

${ }^{31}$ H. Doerk, PhD thesis (2012).

${ }^{32}$ M. Oberparleiter, PhD Thesis (2015).

${ }^{33}$ E. A. Belli and J. Candy, Plasma Physics and Controlled Fusion 54 (2012), 10.1088/0741- 
$3335 / 54 / 1 / 015015$.

${ }^{34}$ N. Bonanomi, C. Angioni, P. Crandall, A. Di Siena, C. Maggi, and P. Schneider, Nuclear Fusion (2019), 10.1088/1741-4326/ab3ecc.

${ }^{35}$ D. Told, F. Jenko, T. Görler, F. J. Casson, and E. Fable, Physics of Plasmas 20, 122312 (2013). 